\title{
Multi-mode Collaborative Communication Technologies in Wireless Sensor Network
}

\author{
Lina Yuan ${ }^{1, a}$, Jing Gong ${ }^{12, \text { b }}$ and Huajun Chen ${ }^{1, c}$ \\ ${ }^{1}$ School of Information Engineering, Tongren University, Guizhou, 554300,China \\ ${ }^{2}$ Institute of Computer Application Technology, Tongren University, Guizhou ,554300,China \\ a893422817@qq.com, b996075565@qq.com, c390832683@qq.com
} Keywords: WSN, lifecycle of the network, energy consumption, optimization, multi-mode
collaborative communication technologies

\begin{abstract}
For wireless sensor network (WSN), this paper is mainly concerned with how to make the longest lifecycle of the network. Because sensor nodes in WSN are usually used to collaborate on a common goal, it focuses on the optimization of energy consumption of the whole network rather than that of a single node in the process of design. This article put forward one strategy to minimize the total energy consumption from the perspective of a network as a whole that belongs to multi-mode collaborative communication technologies in WSN.
\end{abstract}

\section{The model of the system}

In a typical WSN, information collected by the local nodes needs to be sent to a relatively far distance of the central processing unit (CPU).If the transmission distance is far, information will be sent to a relay node firstly, and then based on the multiple hops routing data is transmitted to the destination nodes. The model is given in figure 1 .

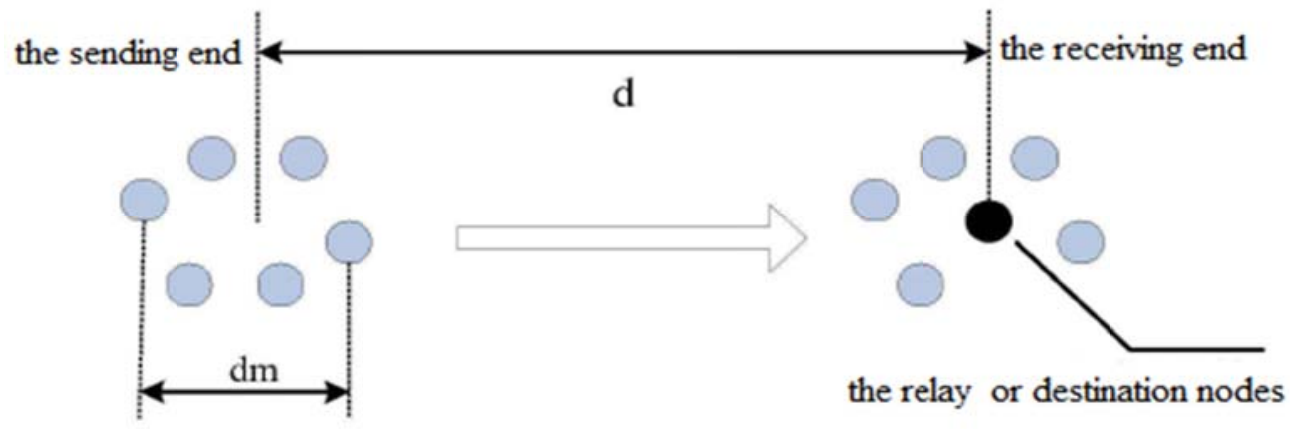

Figure 1 The model of the system

As we all know MIMO technology can save energy in the fading channel. Because the physical size of sensor nodes is smaller, it is not realistic that multiple antennas are directly installed on each node. If multiple nodes are collaborative transmission at the sending end, it can be seen the system as a multiple-antenna system, the equivalent of a MISO system. Using this equivalent MISO system, the needed transmitted energy for long distance transmission can be substantially reduced. In order to make cooperative transmission possible, before transmitting in long distance, it is very necessary to exchange local data. The energy consumption of local data with exchange is less than that of using MISO structure can save. In addition, MISO solutions and the non-collaborative solutions have different parameters with delay. This paper will compare energy efficiency and delay of MISO solutions with that of the non-collaborative solutions, and then select more effective solutions. Using collaborative communication at the sending end is not the only solution to be studied. At the receiving end, multiple nodes can also be deployed around the destination nodes, which make it possible to collaboratively receive. This can also get a MIMO system or a equivalent SIMO system. Similarly, data received by the receiving nodes needs to collect, which also consumes energy. Of course, the total delay will change accordingly. 


\section{Study the performance of the system}

Assuming systems without error correction of coding, if using the technology discussed in ref. [1], the research results of this paper can also be extended to the encoded system. In order to compare the performance of the MIMO solutions with that of the collaborative solutions, assuming there are sending nodes of $\mathrm{M}$, each sending node has $\mathrm{N}$ bits of information to be sent. For the non-collaborative solutions, under the assumption that each sending node uses different time slot to send information to the destination nodes, when sending data, un-coded MQAM is used. For MIMO solutions, at the sending end sending nodes of $M_{t}$ will be collaborative communication, each node first uses different time slot to broadcast information of the local nodes to other nodes in the cluster. After each node receives all information of other nodes, using Alamouti diversity coding ${ }^{[2]}$ edits the sending order of each node. So each node has a predefined number i, all nodes according to the size of the order with $i$ to send information. Similarly, at the receiving end there have $M_{r}$ nodes (including a destination node and $M_{r}-1$ secondary nodes) to collaboratively receive. The $M_{r}-1$ secondary nodes firstly quantize each sign received to $n_{r}$ bits, and then send all bits of information to the destination nodes using un-encoded MQAM. Lastly the destination nodes proceed to unite detection. Because the baseband processing of Alamouti coding is simple ${ }^{[2]}$, for the convenience of calculation, its energy consumption is ignored. Therefore, each node of the total energy consumption includes energy consumption of emission and the circuits. For long distance transmission system, this paper assumes that the transmission channel is square law path attenuation of Rayleigh fading channel. Within the cluster (both transmitter and receiver), suppose the biggest separate distance is $d_{m}$, and assume that each node according to the different transmission distance automatically optimize constellation size. And because the distance of the transmitting terminal and receiving end is $d$ that is far greater than maximum separate distance- $d_{m}$ within the cluster. So when calculating the distance from each node within the emitting cluster to the receiving end, $d_{m}$ will be ignored in this article, assume that the distance from each node within the emitting cluster to the receiving end is $d$.

\section{Study the total energy consumption of the system}

Firstly, the sending end within cluster-heads to broadcast information send energy needed by every bit defined as $E_{i}^{t}, i=1, \ldots, M_{t}$. The receiving end within cluster-heads to broadcast information send energy needed by every bit defined as $E_{j}^{r}, j=1, \ldots, M_{r}-1$. The energy consumption can be calculated according to the known SISO communication with the energy consumption of the formula $^{[3][4]}$ in AWGN channel. Secondly, when transmitting long distance in MIMO systems, the energy needed by every bit of information is defined as $E_{b}^{r}$, and the value of $E_{b}^{r}$ can be calculated according to the formula $E_{b t}=(1+\alpha) \frac{M_{t} N_{0}}{\bar{P}_{b}{ }^{1 / M_{t}}} \times \frac{(4 \pi d)^{2}}{G_{T} G_{R} \lambda^{2}} M_{l} N_{f}+\frac{P_{C}}{R_{b}}$. The non-collaborative traditional transmission solutions are adopted by the long distance transmission of SISO, so the energy needed by transmitting every bit of information is defined as $E_{i}^{0}$, and in the above calculating formula of MIMO system suppose $M_{t}=M_{r}=1$ find out the value of $E_{i}^{0}$.

According to the above assumption, the total energy consumption of non-collaborative traditional transmission solutions can be got: $E_{\text {tra }}=\sum_{i=1}^{M_{t}} N_{i} E_{i}^{0}$

While for the collaborative MIMO solutions, the total energy consumption is divided into three parts: the generated energy consumption of the sending end within the clusters the energy consumption of long distance transmitting nodes and the generated energy consumption of the receiving end within the clusters: $E_{\text {MIMO }}=\sum_{i=1}^{M_{t}} N_{i} E_{i}^{t}+E_{b}^{r} \sum_{i=1}^{M_{t}} N_{i}+\sum_{j=1}^{M_{r}-1} E_{j}^{r} n_{r} N_{s}$ 
In the above formula, $N_{s}=\sum_{i=1}^{M_{t}} N_{i} / b_{m}$ is the numbers of the total signs received by the sending end, and the size constellation is $b_{m}$.

\section{Study the total delay of the system}

In communication systems, the size of the system transmission delay is one of the important criterions to measure their performance. Especially for the limited energy of WSN, if in the process of the whole communication transmission delay is too big, to a certain extent, it will increase energy consumption of sensor nodes, and then affect the efficiency of the whole communication network. And the wide ranges of applications of WSN, which may be in some special applications, have a high requirement of delay in systems. Therefore, the size of delay in systems is also the important direction of research in this paper. This section will study time delay performance of the collaborative MIMO solutions and the non-collaborative traditional transmission solutions. The total transmission delay is defined as the time delay. For a fixed transmission bandwidth $\mathrm{B}$, assuming symbol cycle is approximate for $T_{s} \approx 1 / B$. For the non-collaborative traditional transmission solutions, the total time delay of transmission- $T_{\text {tra }}$ is $\quad T_{\text {tra }}=\sum_{i=1}^{M_{t}} \frac{N_{i}}{b_{i}^{0}} T_{s}$

$b_{i}^{0}$ is the constellation of the i node, and $N_{i} / b_{i}^{0}$ is the numbers of signs sent by the i node. For the collaborative MIMO solutions, the total time delay of $T_{\text {MIMO }}$ includes two parts: the time delay of the local communication at the sending and receiving end and the time delay of long distance transmission.

$T_{\text {MIMO }}=T_{s}\left(\sum_{i=1}^{M_{t}} \frac{N_{i}}{b_{i}^{0}}+\frac{\sum_{i=1}^{M_{t}} N_{i}}{b_{m}}+\sum_{j=1}^{M_{t}-1} \frac{N_{s} n_{r}}{b_{j}^{r}}\right)$

Among $b_{i}^{t}$ and $b_{j}^{r}$ are separately constellation size of the i node within the clusters at the sending end and the $\mathrm{j}$ node within the clusters at the receiving end in the process of local communication.

In the above formula, the first and the third part are separately time delay of local communication at the sending and receiving end, and the second part is time delay generated by the long transmission distance of MIMO.

\section{The simulation experiment}

In this section, assuming that the maximum distance within the clusters is $d_{m}=1 \mathrm{~m}$, the bandwidth of transmission is $B=10 \mathrm{kHz}, n_{r}=10$. The numbers of bits needed by all sending nodes need are the same, $N_{i}=10 \mathrm{~kb}$. It discusses the three transmission solutions-MISO, SIMO, and MIMO in the following.

\section{The transmission plan of MISO}

First consider only the sending end to use collaborative communication. Assuming $M_{t}=2, M_{r}=1$, and other parameters of systems refer to the literature $[3,4]$ to process simulation. 


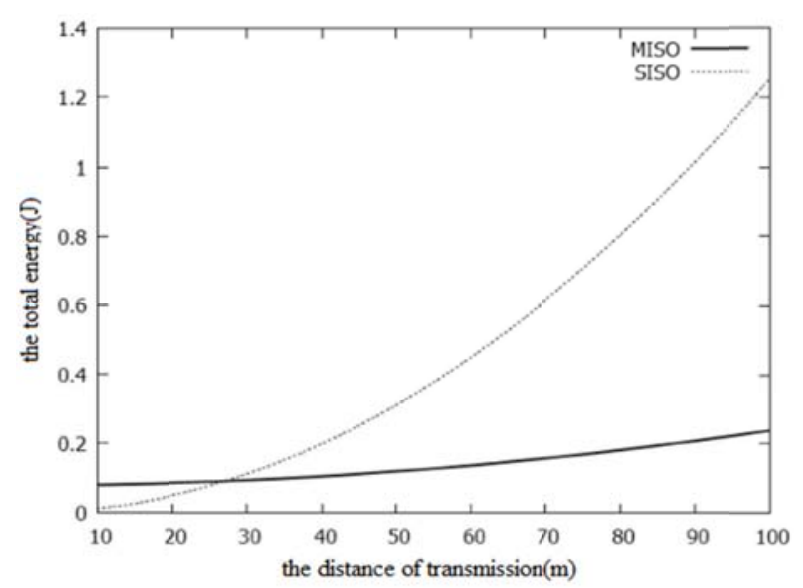

Figure 2 The comparison of energy consumption of MISO solutions with that of SISO solutions

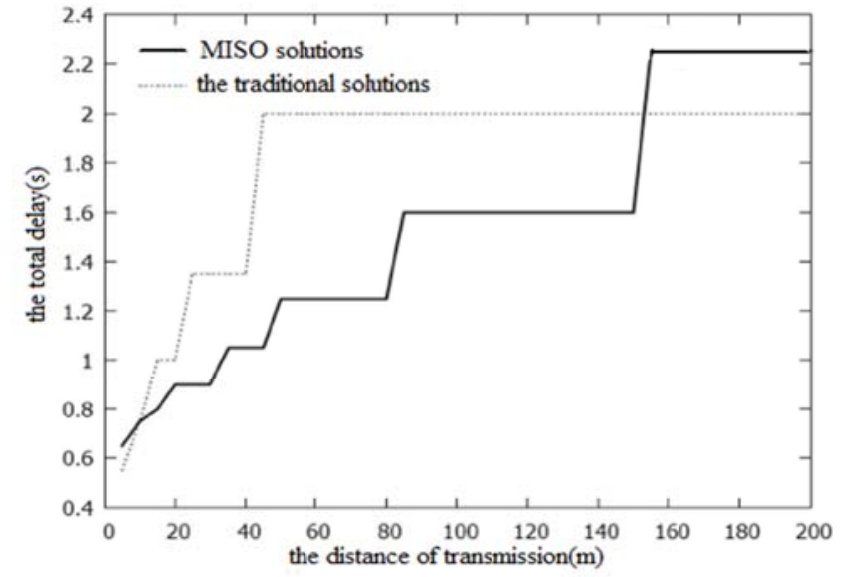

Figure 3 The comparison of delay performance of MISO solutions with that of

the traditional solutions

Figure 2 shows when the distance of transmission is $d \geq 28 m$ in WSN, the total energy efficiency of MISO transmission solutions is higher than the non-collaborative traditional transmission solutions. And with the increase of the transmission distance-d, using MISO transmission solutions can save more and more energy. When $d=100 \mathrm{~m}$, using MISO transmission solutions can save $100 \%$ or so than the non-collaborative traditional transmission solutions.

Figure 3 expresses the comparison of the performance of transmission time delay between MISO transmission solutions and non-collaborative solutions. From the figure 3 to be seen, when the transmission distance is within certain region, the transmission time delay of transmission solutions is less. Because the capacity of the chained path in MISO is greater than in SISO, when the transmission distance is shorter, select the bigger constellation size to reduce energy consumption of circuits, which overcomes the overhead on time delay of local communication at the sending and receiving end in MISO solutions. The transmission time delay of long transmission distance in MISO solutions is less. When the transmission distance continues to increase, the transmission energy becomes the main overhead of the total energy. At present, for the MISO solutions and non-collaborative transmission solutions, the optimal constellation size all becomes $b=2$, which leads to the long distance of transmission in the two solutions as the same. Meanwhile MISO solutions include the additional communication delay brought by the flow of local information, under whose condition, the transmission delay of MISO solutions is greater than the non-collaborative traditional solutions. Moreover, from the above two figures to be known, there exists a region of distance (from $28 \mathrm{~m}$ to $160 \mathrm{~m}$ ), which makes the performance of energy consumption and time delay in MISO solutions still further superior.

\section{The transmission plan of MISO}

In this section consider only the receiving end to use collaborative communication, while the sending end only to one node to work namely $M_{t}=1, M_{r}=2$, and other parameters of systems are the same as the above section. 


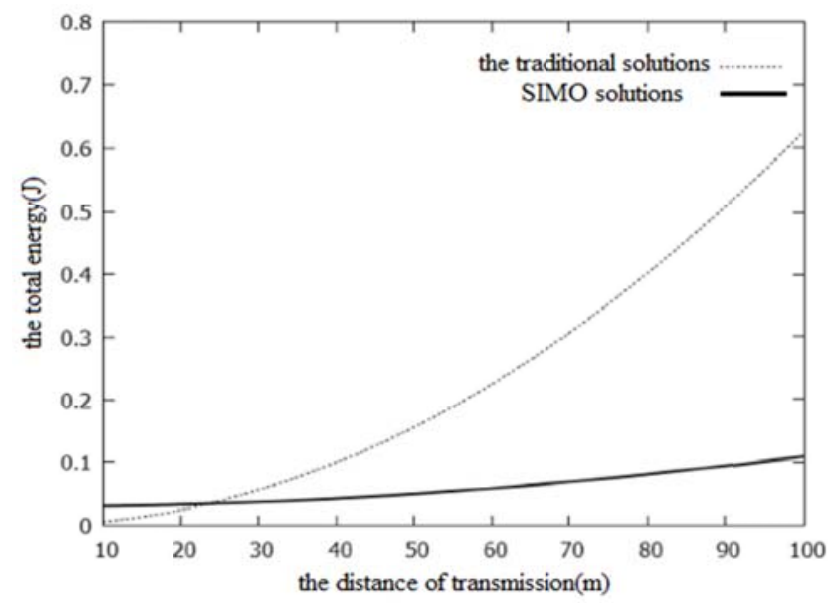

Figure 4 The comparison of energy consumption of SIMO solutions with that of the traditional solutions

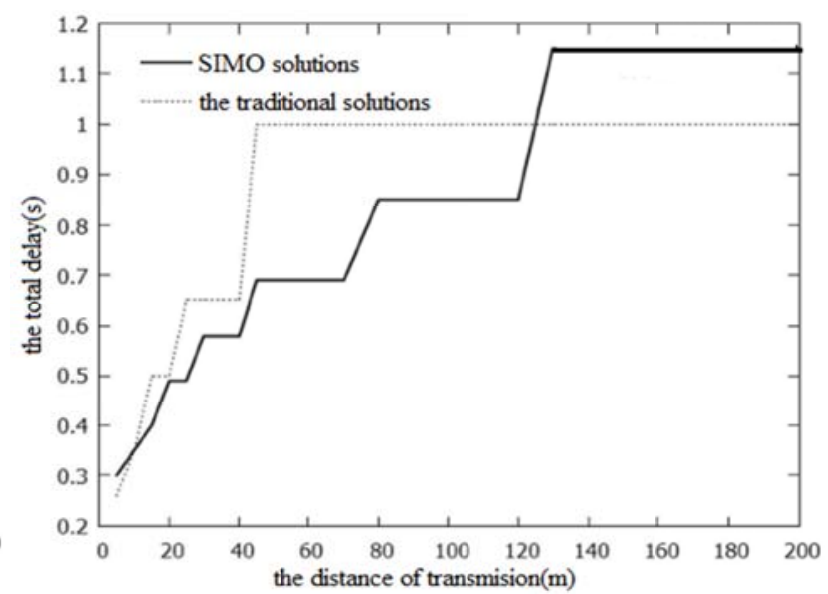

Figure 5 The comparison of delay performance of SIMO solutions with that of the traditional solutions

The figure 4 shows in WSN using SIMO transmission solutions are the same as MISO transmission solutions, and there exists a certain threshold (in this case, the value of threshold$d=24 \mathrm{~m}$ ). With the transmission distance $d \geq 24 \mathrm{~m}$, the total energy efficiency of SIMO transmission solutions is higher than the non-collaborative traditional transmission solutions. And with the increase of the transmission distance d, using SIMO transmission solutions can save more and more energy. With $d=100 \mathrm{~m}$, using SIMO transmission solutions can save $100 \%$ or so energy than the non-collaborative traditional transmission solutions.

The figure 5 compares the delay performance of SIMO solutions with that of non-collaborative traditional transmission solutions. As can be seen from the figure, similar to MISO transmission solutions, when the transmission distance is within a certain region (in this case, $128 \mathrm{~m} \geq d \geq 10 \mathrm{~m}$ ), the delay performance of SIMO solutions is superior to the non-collaborative traditional solutions. As can be known to combine figure 4 with figure 5 , there exists a region (in this case, $128 m \geq d \geq 24 m$ ). Within this region, using SIMO transmission solutions is more effective than non-collaborative traditional solutions either in energy efficiency or in time delay performance.

\section{The transmission plan of MISO}

Lastly, consider using the collaborative communication in MIMO transmission solutions at the sending and receiving end. Assuming that $M_{t}=2, M_{r}=2$, and other parameters of systems are still the same as the above section.
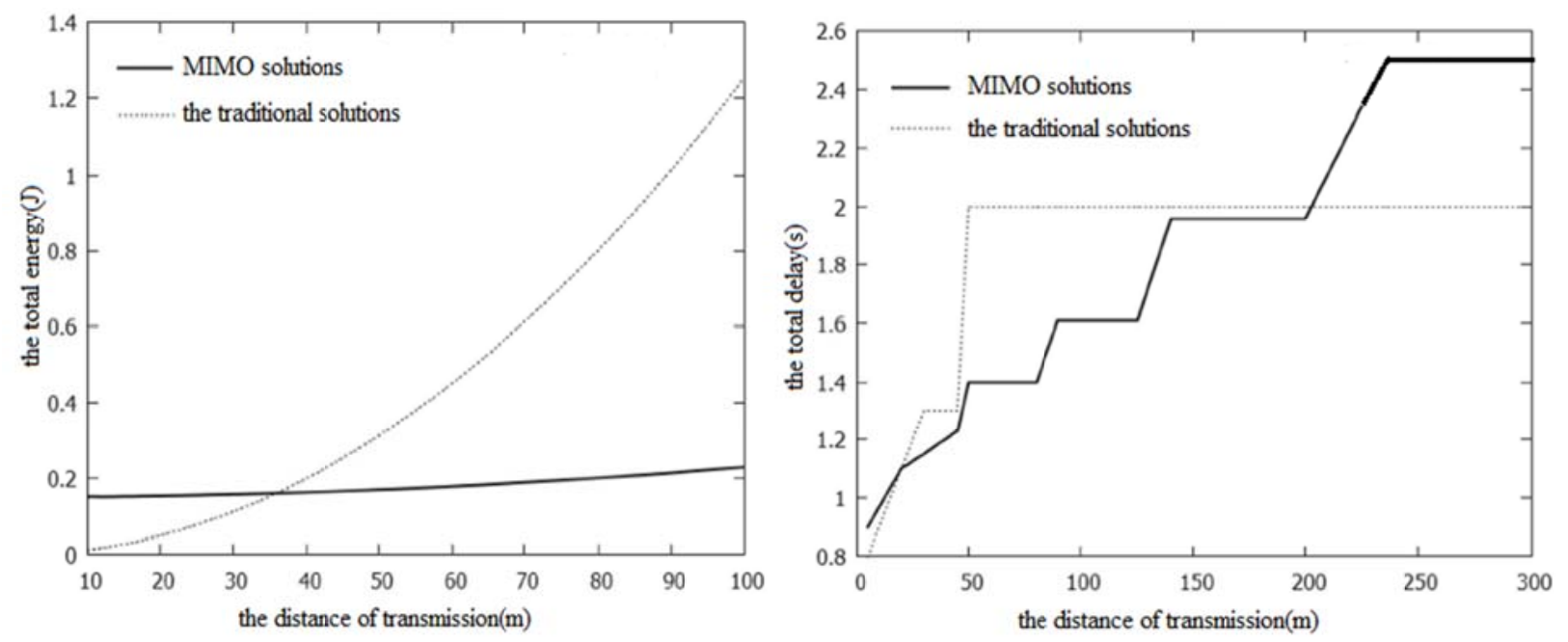

Figure 6 The comparison of energy consumption solutions with that of the traditional solutions

Figure 7 The comparison of delay performance of MIMO of MIMO solutions with that of the traditional solutions

Figure 6 compares the total energy consumption of MIMO solutions with that of non-collaborative solutions with the change of the transmission distance-d. The structure of MIMO is more complex than that of MISO or SIMO, which leads to consume more energy with transmitting local communication in MIMO. The energy efficiency of using MIMO solutions is 
higher than non-collaborative traditional solutions and the threshold of MIMO solutions is greater than that of MISO and SIMO solutions (in this case, the threshold $d=37 \mathrm{~m}$ ). Because energy needed by transmitting long distance in MIMO technologies is less than other transmitting modes, when the transmission distance-d is enough great, the total energy consumption of using MIMO solutions is less than that of MISO and SIMO solutions.

Figure 7 compares the time delay performance of MIMO solutions with that of non-collaborative traditional transmission solutions. Similar to the former two solutions, there exists a region, within which (in this case, the threshold $200 m \geq d \geq 37 \mathrm{~m}$ ), the performances of energy consumption and time delay are all less than other non-collaborative solutions.

\section{Summary}

This paper firstly puts forward using system model of the collaborative MIMO technologies between nodes in WSN, and briefly introduces the working principle of collaborative communication between nodes: in order to make it possible to cooperative transmission, before transmission in long distance processing local data to exchange is very necessary. Then the overall performance of the collaborative MIMO communication systems is analyzed, and the calculation formulas and methods about the total energy consumption and time delay of collaborative MIMO systems and the non-collaborative traditional transmission systems are given. Finally on the basis of the above all contents, through the actual data, respectively compare energy efficiency and delay performance of the non- collaborative traditional transmission solutions with that of MISO transmission solutions that only the sending end uses the collaborative communication, SIMO transmission solutions that only the receiving end uses the collaborative communication, and as well as MIMO transmission solutions that the sending and receiving end all use collaborative communication technology.

The simulation results show that there must be an interval, when the transmission distance of systems is in this range of change over time, energy efficiency and delay performance of all the above three transmission solutions will be all superior to the non-collaborative traditional transmission solutions at the same time.

\section{Acknowledgment}

Serial Number: The Collaborative Fund Project of Science and Technology Agency in Guizhou Province Marked by the word LH on 7487[2014], and the reform project of teaching contents and curriculum system in colleges and universities of Guizhou Province on 2014SJJGXM003.

Sustentation Fund : The Collaborative Fund Project of Science and Technology Agency, and the education office in Guizhou Province.

\section{References}

[1] Energy-constrained modulation optimization for coded systems[C]. in Proc. GLOBECOM'03, SanFrancisco,CA,Dec.2003,pp.372-376.[Online].Available: http://wsl.stanford.edu/Publications.

[2]A. Paulraj, R. Nabar, D. Gore. Introduction to Space-Time Wireless Communications[M], Cambridge, U.K.:Cambridge Univ. Press, 2003.preprint.

[3] S. Cui, A. J. Goldsmith, A. Bahai. Energy-constrained modulation optimization [J]. IEEE Trans. Wireless Commun., 2004. [Online]. Available: http://wsl.stanford.edu/Publications.html, submitted for publication.

[4] S.Cui, A. J. Goldsmith, A. Bahai. Modulation optimization under energy constraints[C]. inProc. ICC'03,AK,May2003,pp.2805-2811. [Online]. Available: http://wsl.stanford.edu/ Publications.html. [5]ZHANG GuoPeng,Pareto optimal time-frequency resource allocation for selfish wireless 
cooperative multicast networks[J]. Science China(Information Sciences), December 2013, Vol. 56 122306:1-122306:8

[6]S.Chu,X.Wang, Y.Yang, Adaptive scheduling in MIMO-based heterogeneous ad hoc networks[J], IEEE Trans.Mob.Comput.13(5)(2014)964-978.

[7]Lina Yuan, Jing Gong,An Analysis of Large-scale Wireless Sensor Networks with Energy-saving Problem[J].Applied Mechanics and Materials, Vol 686 (2014) p.412-416. 